\title{
Reflecting a Triangle in the Plane
}

\author{
Imre Bárány, ${ }^{1}$ Peter Frankl ${ }^{2}$ and, Hiroshi Maehara ${ }^{3}$ \\ 1 Yale University, New York University, and the Mathematical Institute of the \\ Hungarian Academy of Sciences \\ 2 CNRS, Paris, France \\ ${ }^{3}$ Department of Mathematics, Faculty of Education, Ryukyu University, Nishihara-cho, \\ Okinawa, 903-01 Japan
}

\begin{abstract}
We prove that if the three angles of a triangle $T$ in the plane are different from $\left(60^{\circ}, 60^{\circ}, 60^{\circ}\right),\left(30^{\circ}, 30^{\circ}, 120^{\circ}\right),\left(45^{\circ}, 45^{\circ}, 90^{\circ}\right),\left(30^{\circ}, 60^{\circ}, 90^{\circ}\right)$, then the set of vertices of those triangles which are obtained from $T$ by repeating 'edge-reflection' is everywhere dense in the plane.
\end{abstract}

\section{Introduction}

An edge-reflection of a triangle $T_{1}$ is a triangle $T_{2}$ which is symmetric to $T_{1}$ with respect to the line determined by an edge of $T_{1}$ (see Fig. 1). By a chain of triangles we mean a sequence of triangles

$$
T_{1}, T_{2}, T_{3}, \ldots
$$

such that $T_{i}(i \geq 2)$ is an edge-reflection of $T_{i-1}$, and $T_{i} \neq T_{i-2}$ for $i \geq 3$. Two triangles $A B C$ and $P Q R$ are equivalent to each other if $A B C=P Q R$ or there is a finite chain of triangles $T_{1}, \ldots, T_{n}$ such that $T_{1}=A B C$ and $T_{n}=P Q R$. This is clearly an equivalence relation.

Let us denote by $\Omega_{A B C}$ (or simply by $\Omega$ ) the set of vertices of the triangles equivalent to a given triangle $A B C$. Figure 2 shows part of $\Omega$ for four types of triangles with angles

$$
\begin{aligned}
& \left(60^{\circ}, 60^{\circ}, 60^{\circ}\right),\left(30^{\circ}, 30^{\circ}, 120^{\circ}\right), \\
& \left(45^{\circ}, 45^{\circ}, 90^{\circ}\right),\left(30^{\circ}, 60^{\circ}, 90^{\circ}\right) .
\end{aligned}
$$

We are going to prove that except for the above four types of triangles, $\Omega$ is everywhere dense in the plane (Theorems 2 and 3).

\section{The Angles of a Triangle}

In this paper all angles are measured by degree $\left({ }^{\circ}\right)$. A triangle $A B C$ is called rational if its three angles are all rational angles, otherwise, $A B C$ is called irrational. It is obvious that if $A B C$ is irrational, then at least two angles are irrational. 


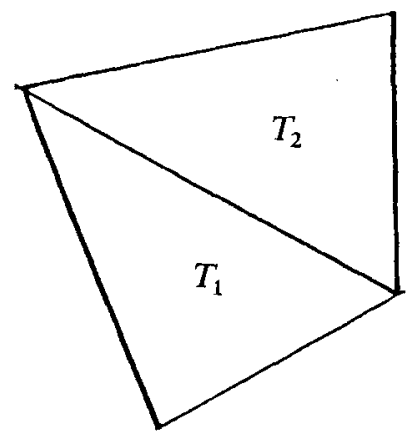

Fig. 1

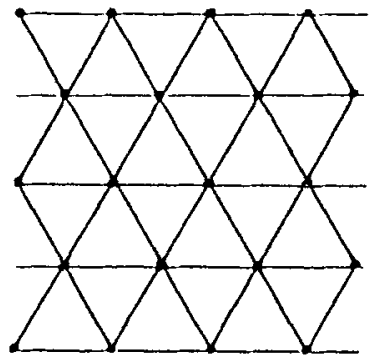

$\left(60^{\circ}, 60^{\circ}, 60^{\circ}\right)$

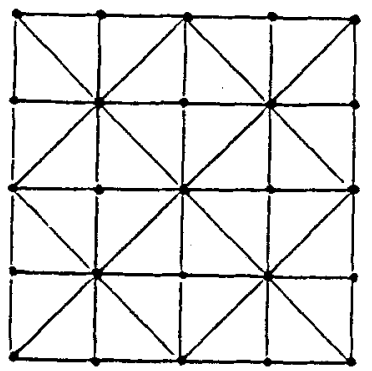

$\left(45^{\circ}, 45^{\circ}, 90^{\circ}\right)$

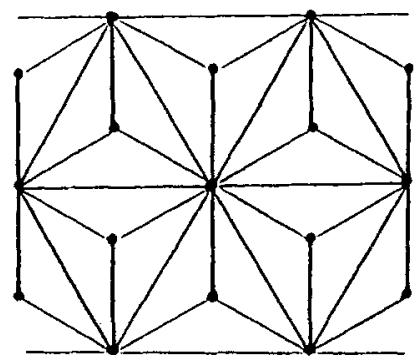

$\left(30^{\circ}, 30^{\circ}, 120^{\circ}\right)$

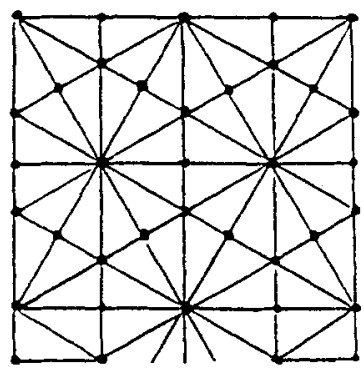

$\left(30^{\circ}, 60^{\circ}, 90^{\circ}\right)$

Fig. 2 
Let $\alpha$ be a rational angle and $m / n$ be the irreducible fraction equatl to $\alpha / 180^{\circ}$. If $m$ is even, then the angle $\alpha$ is called even-type, otherwise it is called odd-type. Further, an odd-type angle is called (odd/odd)-type or (odd/even)-type accordingly as the denominator of the irreducible fraction is odd or even. For example, $30^{\circ}$ is (odd/even)type and $60^{\circ}$ is (odd/odd)-type. If $2 \alpha$ is odd-type, then clearly $\alpha$ is (odd/even)-type.

Theorem 1. Among the three angles $\alpha, \beta, \gamma$ of a rational triangle $A B C$ :

(1) At least one angle is odd-type.

(2) The number of (odd/even)-type angles is $\neq 1$.

(3) The number of (odd/odd)-type angles is $\neq 2$.

Proof. First suppose that the three angles $\alpha, \beta, \gamma$ are all even-type. Then since $\alpha / 180^{\circ}+\beta / 180^{\circ}+\gamma / 180^{\circ}=1$, we have

$$
\text { even/odd }+ \text { even/odd }+ \text { even/odd }=1
$$

which implies even + even + even $=$ odd, a contradiction. Thus (1) follows.

Next, suppose that $\alpha$ is (odd/even)-type, but $\beta, \gamma$ are not. Then, from $2 \alpha / 180^{\circ}+$ $2 \beta / 180^{\circ}+2 \gamma / 180^{\circ}=2$, we have

$$
\text { odd } / n+\text { even/odd }+ \text { even/odd }=2
$$

which implies odd $=n \cdot$ even, a contradiction. Thus (2) follows.

Finally, suppose $\alpha, \beta$ are (odd/odd)-type, but $\gamma$ is even-type. Then we have

$$
\text { odd/odd }+ \text { odd/odd }+ \text { even/odd }=1
$$

which implies that even $=$ odd + odd + odd, a contradiction. Thus $(3)$ follows.

Corollary 1. In a rational triangle $A B C$, one of the following three cases occurs:

(1) Two or three angles are (odd/even)-type.

(2) One angle is (odd/odd)-type, and the other two are even-type.

(3) Three angles are (odd/odd)-type.

\section{Rational Triangles}

Lemma 1. Let $A B C$ be a rational triangle. Let $A B^{\prime} C^{\prime}$ be the triangle symmetric to $A B C$ with respect to the point $A$, and let $A B^{\prime \prime} C^{\prime \prime}$ be the triangle symmetric to $A B^{\prime} C^{\prime}$ with respect to the bisector of the angle $\angle A=\alpha$ (see Fig. 3):

(1) If $\alpha$ is (odd/even)-type, then $A B^{\prime} C^{\prime}$ is equivalent to $A B C$.

(2) If $\alpha$ is (odd/odd)-type, then $A B^{\prime \prime} C^{\prime \prime}$ is equivalent to $A B C$.

(3) If $\alpha$ is even-type, then $A B^{\prime \prime} C^{\prime \prime}$ is equivalent to $A B^{\prime} C^{\prime}$.

Proof. Suppose $\alpha$ is (odd/even)-type, i.e., $\alpha / 180^{\circ}=(2 m+1) /(2 n)$. Then $2 n \alpha=$ $(2 m+1) 180^{\circ} \equiv 180^{\circ}\left(\bmod 360^{\circ}\right)$. Hence, in a chain of triangles

$$
A B C, A B C_{1}, A B_{1} C_{1}, A B_{1} C_{2}, A B_{2} C_{2}, \ldots, A B_{n} C_{n}
$$




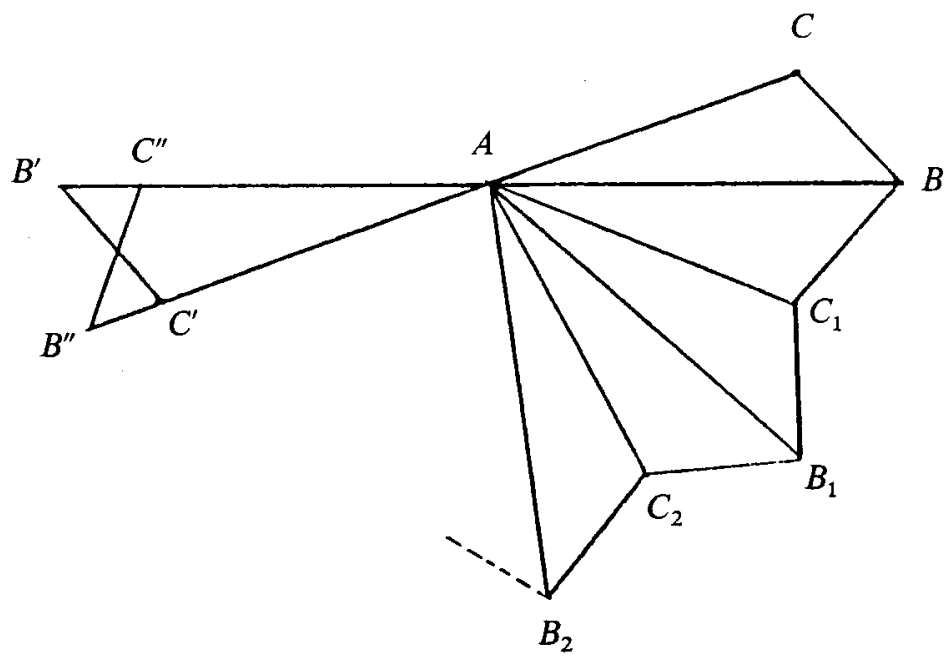

Fig. 3

with common vertex $A$ (Fig. 3), the last triangle $A B_{n} C_{n}$ will coincide with $A B^{\prime} C^{\prime}$ or $A B^{\prime \prime} C^{\prime \prime}$. However, since an even number of edge-reflections results in a congruent triangle of the same 'sense', we must have $A B_{n} C_{n}=A B^{\prime} C^{\prime}$. This proves (1). Similarly, we can get (2) (3).

If $\theta=60^{\circ}, 90^{\circ}, 120^{\circ}$, or $180^{\circ}$, then $\cos \theta=1 / 2,0,-1 / 2$, or -1 . And it is well known that if $|\cos \theta|$ is a rational number other than $0,1,1 / 2$, then $\theta$ is irrational (see, e.g., Hadwiger-Debrunner [1], problem 8).

Lemma 2. Let $\theta\left(0^{\circ}<\theta \leq 180^{\circ}\right)$ be a rational angle. Then $\cos \theta$ is a rational number if and only if $\theta=60^{\circ}, 90^{\circ}, 120^{\circ}$, or $180^{\circ}$.

Lemma 3. For a given angle $\alpha$ and a real number $r>0$, let $\Lambda(r, \alpha)$ denote the set of all points represented by linear combinations of plane-vectors

$$
(r \cdot \cos k \alpha, r \cdot \sin k \alpha), \quad k=-1,0,1,2,
$$

with integral coefficients. If $\cos \alpha$ is irrational, then $\Lambda(r, \alpha)$ is everywhere dense in the plane.

Proof. Suppose that $\lambda:=2 \cos \alpha$ is irrational. Since

$$
(r \cdot \cos \alpha, r \cdot \sin \alpha)+(r \cdot \cos (-\alpha), r \cdot \sin (-\alpha))=(\lambda r, 0)
$$

$\Lambda(r, \alpha)$ contains

$$
\{m(\lambda r, 0)+n(r, 0): m, n \in Z\}
$$

Hence the closure $\bar{\Lambda}(r, \alpha)$ of $\Lambda(r, \alpha)$ contains the $x$-axis. Similarly, $\bar{A}(r, \alpha)$ contains the line determined by the vector $(\cos \alpha, \sin \alpha)$. Further, since $A(r, \alpha)$ is closed under the addition, we have the lemma.

In the rest of this section, let $A B C$ be a rational triangle with vertex $A$ at the 
origin. We use the same notation $A(r, \alpha)$ as in Lemma 3 . The angles and edges of the triangle $A B C$ are denoted by $\alpha, \beta, \gamma$ and $a, b, c$, as usual.

Lemma 4. If $\alpha, \beta$ are (odd/even)-type, then $\Omega$ contains $\Lambda(2 c, 2 \alpha)$. Hence, if $\alpha \neq 30^{\circ}$, $45^{\circ}, 90^{\circ}$, then $\Omega$ is dense in the plane.

Proof. From Lemma 1(1) and Fig. 4, it follows that $\Omega$ contains $\Lambda(2 c, 2 \alpha)$. The latter part follows from Lemmas 2,3 and $\alpha \neq 60^{\circ}$.

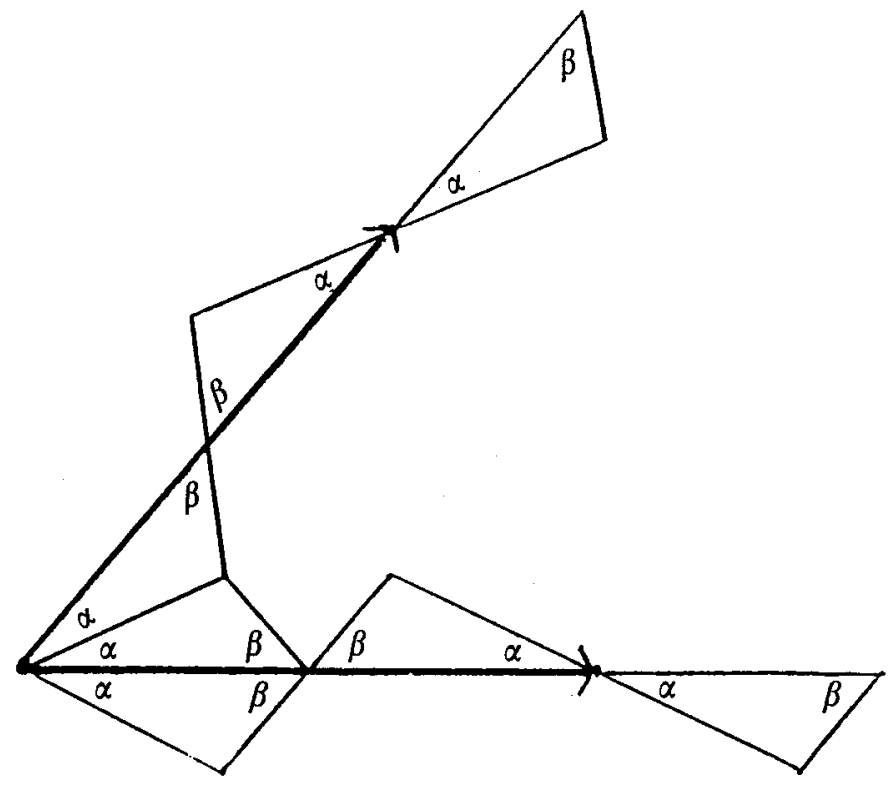

Fig. 4

Lemma 5. If $\alpha, \beta, \gamma$ are all (odd/odd)-type, then $\Omega$ contains $A(a+b+c, \alpha)$. Hence, if $\alpha \neq 60^{\circ}$, then $\Omega$ is dense in the plane.

Proof. From Lemma 1(2) and Fig. 5, $\Omega$ contains $A(a+b+c, \alpha)$. The latter part follows from Lemmas 2, 3 .

Lemma 6. If $\alpha, \beta$ are even-type and $\gamma$ is (odd/odd)-type, then $\Omega$ contains $\Lambda(a+b-c, \alpha)$. Hence $\Omega$ is dense in the plane.

Proof. From Lemma 1(2), (3) and Fig. 6, $\Omega$ contains $A(a+b-c, \alpha)$. Since one of $\alpha$, $\beta$ is less than $120^{\circ}$, the latter part follows from Lemmas 2,3 .

Theorem 2. Let $A B C$ be a rational triangle with angles $\alpha \leq \beta \leq \gamma$. If

$$
\begin{gathered}
(\alpha, \beta, \gamma) \neq\left(60^{\circ}, 60^{\circ}, 60^{\circ}\right), \quad\left(30^{\circ}, 30^{\circ}, 120^{\circ}\right) \\
\left(45^{\circ}, 45^{\circ}, 90^{\circ}\right), \quad\left(30^{\circ}, 60^{\circ}, 90^{\circ}\right)
\end{gathered}
$$

then $\Omega_{A B C}$ is everywhere dense in the plane. 


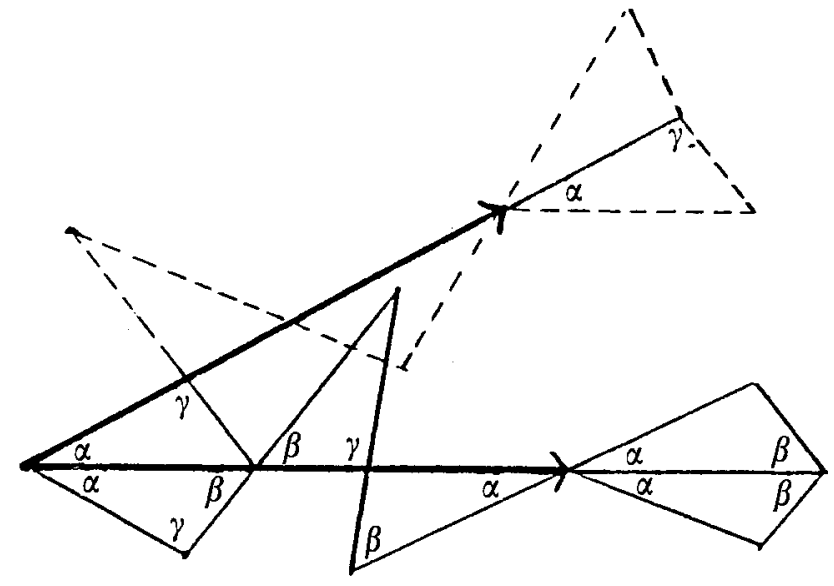

Fig. 5

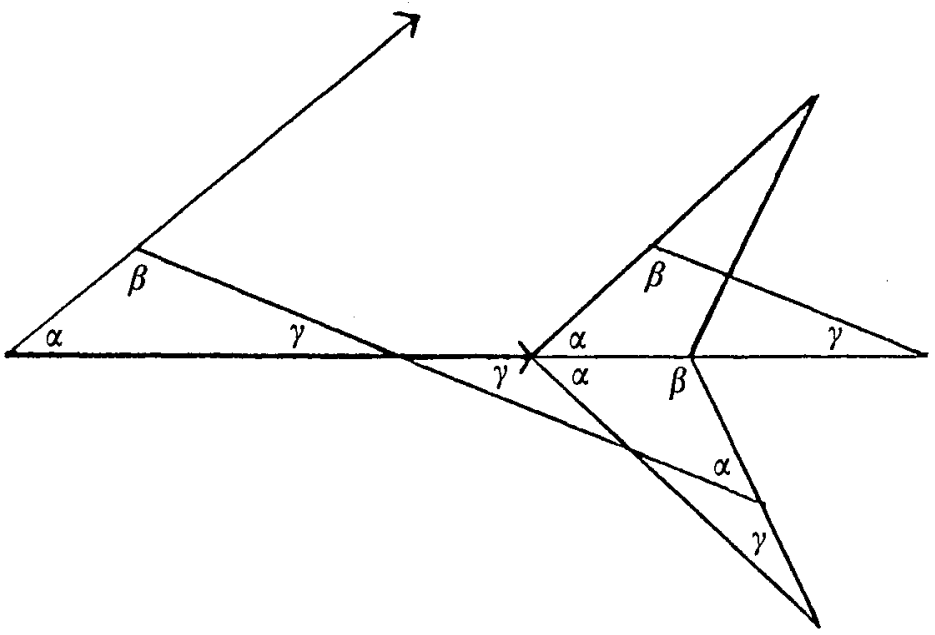

Fig. 6

Proof. Let us consider the three cases (1), (2), and (3) of Corollary 1.

Case (1). If $A B C$ has two (odd/even)-type angles different from $30^{\circ}, 45^{\circ}, 90^{\circ}$, then $\Omega$ is dense in the plane by Lemma 4 . If the two angles are $30^{\circ}, 45^{\circ}$, then the third angle is $105^{\circ}$, which is (odd/even)-type. Hence, unless $(\alpha, \beta, \gamma)=\left(30^{\circ}, 30^{\circ}, 120^{\circ}\right)$, $\left(45^{\circ}, 45^{\circ}, 90^{\circ}\right), \Omega$ is dense in this case.

Case (2). If $A B C$ has one (odd/odd)-type angle and two even-type angles then $\Omega$ is dense in the plane by Lemma 6 .

Case (3). If $A B C$ has three (odd/odd)-type angles, and $A B C$ is not equilateral, then $\Omega$ is dense in the plane by Lemma 5 . 


\section{Irrational Triangles}

The following lemma will be obvious.

Lemma 7. Let

$$
P Q R, P Q R_{1}, P Q_{1} R_{1}, P Q_{1} R_{2}, P Q_{2} R_{2}, \ldots
$$

be an infinite chain of triangles with common vertex $P$. Then:

(1) for any point $X \neq P$, there exists an $n$ such that

$$
\min \left(\angle X P Q_{n}, \angle X P R_{n}\right) \leq 60^{\circ}
$$

and

(2) if $\angle Q P R$ is irrational, then the closures of the sets

$$
\left\{Q, Q_{1}, Q_{2}, Q_{3}, \ldots\right\} \text { and }\left\{R, R_{1}, R_{2}, R_{3}, \ldots\right\}
$$

are concentric circles with center $P$.

Lemma 8. For any irrational triangle $P Q R$ and a point $Y \neq P$, there is a triangle $P U V$ equivalent to $P Q R$ with irrational angle $\angle U$ and $30^{\circ} \leq \angle Y P U \leq 150^{\circ}$.

Proof. Note that an irrational triangle has at least two irrational angles. Hence, if $\angle Q P R$ is irrational then the lemma follows from Lemma $7(2)$. In the case $\angle P$ rational, the two angles $\angle Q, \angle R$ are both irrational, whence, applying Lemma 7(1) for a point $X$ such that $\angle X P Y=90^{\circ}$, we have the lemma.

Theorem 3. If $A B C$ is an irrational triangle, then $\Omega_{A B C}$ is everywhere dense in the plane.

Proof. Suppose there is a point $Y$ in the plane for which

$$
d:=\inf \{|Y-W|: W \in \Omega\}
$$

is positive. Then for any $\varepsilon>0$, there is a triangle $P Q R$ which is equivalent to $A B C$ and

$$
|P-Y|<d+\varepsilon .
$$

By Lemma 8, we may always suppose that $\angle Q$ is irrational and $30^{\circ} \leq \angle Y P Q \leq$ $150^{\circ}$. Hence, if $\varepsilon$ is sufficiently small, the circle with center $Q$ and radius $P Q$ cuts the circle with center $Y$ and radius $d$. Therefore, by Lemma $7(2)$, there is a point $P^{\prime}$ of $\Omega$ with distance $<d$ from $Y$, a contradiction.

\section{Remarks}

Remark 1. For a triangle $T=A B C$, let $\Phi(T)$ denote the set of triangles obtained from $T$ by repeated reflections. Describing a triangle $T^{\prime} \in \Phi(T)$ by its vertex $A^{\prime} \in R^{2}$, the angle of side $A^{\prime} B^{\prime}$ and $x$-axis, and its orientation, we can identify $\Phi(T)$ with a set in $R^{2} \times[0,360) \times\{-1,1\}$. Our result asserts.that except for the four exceptional cases, the canonical projection of $\Phi(T)$ into $R^{2}$ is everywhere dense in $R^{2}$. 
The same proof gives the following: If $T$ is irrational, then $\Phi(T)$ is everywhere dense in $R^{2} \times[0,360) \times\{-1,1\}$. If $T$ is rational, then the canonical projection of $\Phi(T)$ onto $[0,360)$ takes only finitely many values, say $\alpha_{1}, \alpha_{2}, \ldots, \alpha_{n}$. Further, except for the four exceptional cases, $\Phi(T) \cap\left(R^{2} \times\left\{\alpha_{i}\right\} \times\{-1,1\}\right)$ is everywhere dense in $R^{2} \times\left\{\alpha_{i}\right\} \times\{-1,1\}$ for every $i$.

Remark 2. In [2], Laczkovich studied the problem of tiling polygons with similar triangles. A triangle $T$ is said to tile the polygon $P$, if $P$ can be decomposed into finitely many non-overlapping triangles similar to $T$. Among others, he proved that, except right triangles, only three types of triangles with angles

$$
\left(22.5^{\circ}, 45^{\circ}, 112.5^{\circ}\right), \quad\left(45^{\circ}, 60^{\circ}, 75^{\circ}\right) \quad \text { or } \quad\left(15^{\circ}, 45^{\circ}, 120^{\circ}\right)
$$

can tile the square. Further, among rational right triangles, only two types of triangles with angles $\left(45^{\circ}, 45^{\circ}, 90^{\circ}\right),\left(15^{\circ}, 75^{\circ}, 90^{\circ}\right)$ can tile the square.

Remark 3. A sequence of (at least two) congruent regular tetrahedra in $R^{3}$ is called a tetrahedral snake if two consecutive tetrahedra share exactly one face, and every three consecutive tetrahedra are distinct. In 1956, Steinhaus posed the question: In a tetrahedral snake of finite length, can the last tetrahedron be a translation of the first one? This problem was solved negatively by Swierczkowski (see Wagon [4], p. 68).

It was proved in [3] that the set of those points which are obtained as the vertices of tetrahedra in tetrahedral snakes starting from a fixed regular tetrahedron is everywhere dense in the space. Analogous results hold in any dimension $n \geq 3$.

Acknowledgment. The authors would like to thank the referee who pointed out that our result can also be proved by group theoretic arguments.

\section{References}

1. Hadwiger, H., Debrunner, H.: Combinatorial geometry in the plane. New York: Holt, Reinhart and Winston 1964

2. Laczkovich, M.: Tiling of polygons with similar triangles. Combinatorica 10, 281-306 (1990)

3. Maehara, H.: Extending a flexible unit-bar framework to a rigid one. Ann. Discrete Math.

4. Wagon, S.: The Banach-Tarski Paradox Cambridge: Cambridge University Press (1985) 